\title{
ATAQUE POR SULFATO EM CONCRETOS POR MEIO DE ENSAIOS ACELERADOS E MODELAGEM: UMA REVISÃO DA LITERATURA RECENTE
}

\author{
DIAS, ROBERTO L. \\ Engenheiro Civil, mestrando do PPGECC \\ Universidade Federal do Paraná \\ Paraná; Brasil \\ robertoluizdias@hotmail.com
}

MEDEIROS-JUNIOR, RONALDO A.

Professor Doutor

Universidade Federal do Paraná

Paraná; Brasil

medeirosjunior.ufpr@gmail.com

\author{
NENEVÊ, BRUNO L. \\ Engenheiro Civil, mestrando do PPGECC \\ Universidade Federal do Paraná \\ Paraná; Brasil \\ eng.neneve@gmail.com
}

\section{RESUMO}

O estudo do comportamento do concreto submetido ao ataque por sulfatos se dá principalmente por meio de ensaios acelerados, devido as suas vantagens, dentre as quais se destaca a rapidez para obtenção de resultados. Assim, existe na literatura um número expressivo de trabalhos que procuram aperfeiçoar essa técnica. Isto posto, o presente artigo apresenta uma revisão da literatura recente sobre os estudos relacionados ao ataque por sulfatos às matrizes cimentícias. Para tal, foram selecionados 17 artigos internacionais sobre o ataque por sulfato, tanto externo como interno, e os respectivos métodos de ensaio acelerados e modelagens, dos quais discorreu-se mais profundamente sobre 6 . Os resultados mostram que há diversos fatores que influenciam o ataque por sulfatos, tornando a modelagem um tanto complexa. Contudo, estudos recentes mostram que é possível adquirir, por meio dos modelos e ensaios acelerados, resultados condizentes com o ocorrido em realidade

Palavras-chave: ataque por sulfato, reação expansiva, ensaios acelerados, modelagem; revisão da literatura.

\section{ABSTRACT}

The study of the concrete behavior under the sulfate attack occurs mainly through accelerated tests. Therefore, a large number of papers can be founded in the literature. Regarding the modeling of the sulfate attack, several models are present in the literature for the external attack. For the internal attack, the number of models is reduced due its complexity. This paper presents a review of the recent literature about sulfate attack studies. Therefore, 17 international articles were selected about the sulfate attack, external and internal, and their respective accelerated test methods and modeling, 6 of which were further discussed. The results show that there are several factors that influence the sulfate attack (intrinsic and external to the material), making the modeling somewhat complex. However, recent studies shown that it is possible to obtain, by accelerated models and tests, consistent results, according with occurred in reality.

Keywords: sulfate attack, expansive reaction, accelerated testing, modeling, literature review.

\section{INTRODUÇÃO}

Os sulfatos são considerados uns dos agentes químicos mais agressivos às matrizes cimentícias, podendo ser encontrados em diversas formas na natureza. A degradação do concreto devido ao ataque por sulfatos ocorre através de reações químicas destes íons $\left(\mathrm{SO}_{4}{ }^{2-}\right)$ com os compostos hidratados do cimento Portland e podem se manifestar de duas formas distintas: expansão associada à fissuração ou diminuição progressiva da resistência vinculada à perda de massa. No primeiro caso, após o início do processo de fissuração, os sulfatos tendem a penetrar com maior facilidade no interior do material, acelerando ainda mais o processo de deterioração. Já para o segundo caso, ocorre a perda de coesão dos produtos de hidratação do cimento que resultam na degradação da matriz cimentícia (MEHTA e MONTEIRO, 2014).

Quanto à classificação, o ataque por sulfato pode ser dividido, de acordo com a forma de manifestação, em: ataque externo e ataque interno. Quanto ao reagente químico no ataque externo, os três principais são: sulfato de cálcio $\left(\mathrm{CaSO}_{4}\right)$; sulfato de sódio $\left(\mathrm{Na}_{2} \mathrm{SO}_{4}\right)$; e sulfato de magnésio $\left(\mathrm{MgSO}_{4}\right)$, sendo este último considerado o mais agressivo 
(COUTINHO, 2001; HOPPE FILHO, 2015). No que diz respeito ao ataque interno, a pirita é um dos contaminantes mais estudados entre os agregados.

Assim, o estudo sobre tal fenômeno é de significativa importância quanto ao desempenho e à durabilidade dos concretos empregados na construção civil, que vêm apresentando diversos problemas relacionados à degradação precoce. A Figura 1 ilustra o crescimento das pesquisas neste tema, apresentando os artigos registrados internacionalmente nos últimos anos acerca do tema: ataque por sulfatos em concretos.

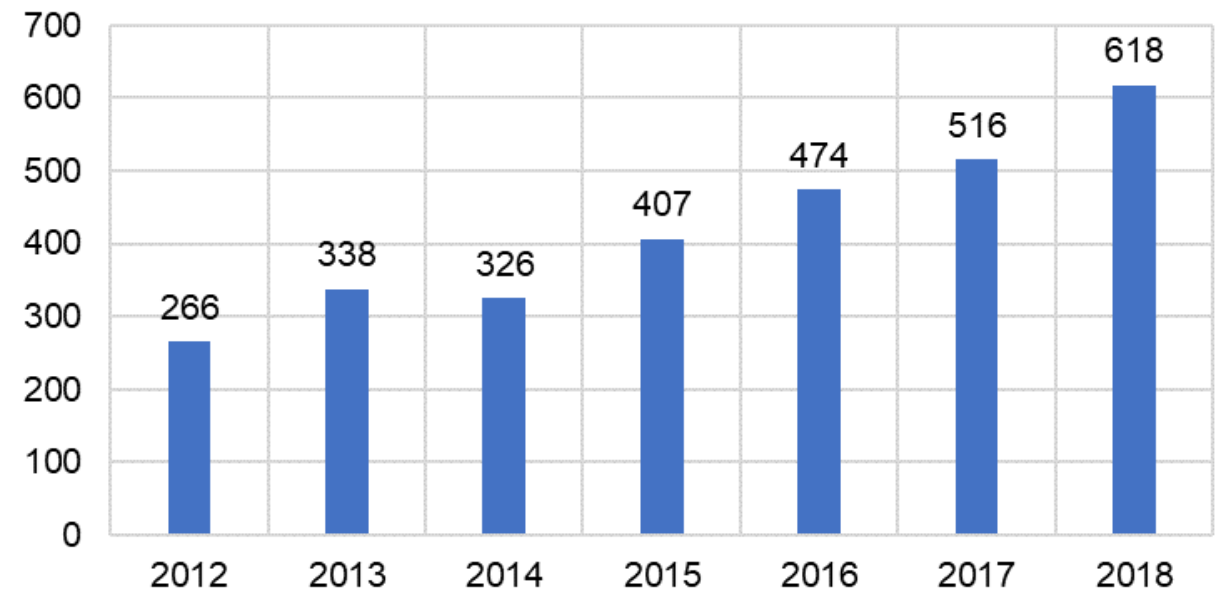

Figura 1 - Artigos publicados relacionados à Ataque por Sulfatos em Concreto (Adaptado de ScienceDirect, 2019)

Isto posto, o presente artigo visa apresentar uma revisão da literatura recente sobre os estudos relacionados ao ataque por sulfatos às matrizes cimentícias, com ênfase em estruturas de concreto. Para tal, serão abrangidos os ataques externos ou internos decorrentes de tal fenômeno, com o estado da arte de métodos de ensaio acelerados e modelagens.

\section{METODOLOGIA}

O trabalho desenvolvido seguiu preceitos do estudo exploratório, por meio de uma pesquisa bibliográfica, desenvolvida a partir de material já elaborado, constituído de artigos científicos. A seguir estão descritas as fontes que forneceram as respostas adequadas para o estudo, na área de Engenharia Civil, sobre ataque por sulfatos em concretos, tanto internos como externos, com abordagem sobre modelagem e ensaios acelerados.

A seleção de artigos se restringiu aos qualis A1 (conforme classificação da CAPES), disponíveis online na plataforma Science Direct (Elsevier), publicados no período de 2012 a 2018. Para busca dos artigos, as palavras-chaves utilizadas, em inglês, foram: Sulfate Attack; External Sulfate Attack; Internal Sulfate Attack; Modelling; Accelerated Test. A coleta de dados, de modo a selecionar e filtrar o material encontrado nas pesquisas, seguiu as seguintes premissas:

- Leitura exploratória de todo o material selecionado, realizada rapidamente, objetivando verificar superficialmente - o enquadramento dentro do escopo do artigo;

- $\quad$ Leitura seletiva, realizada de maneira mais aprofundada nos materiais previamente selecionados;

- Registro das informações extraídas das fontes de instrumento específico (autores, ano, método, resultados e conclusões).

Para análise e interpretação dos resultados obtidos, foi realizada uma leitura analítica com a finalidade de ordenar e sumariar as informações contidas nas fontes, de forma que estas possibilitassem a obtenção de respostas à pesquisa. Por fim, em posse dos artigos selecionados, conforme os critérios supracitados, discorreu-se sobre alguns estudos, de acordo com a relevância do estudo, totalizando 6 artigos comentados com maior especificidade.

\section{RESULTADOS E DISCUSSÕES}

\subsection{Ensaios acelerados}

A NBR 13583 (ABNT, 2014) estabelece o método para determinação da variação dimensional de barras de argamassa de cimento Portland expostas à solução de sulfato de sódio, onde contém todos os parâmetros para realização do ensaio 
- dentro do marco nacional. Porém, novos procedimentos de ensaios acelerados são apresentados frequentemente na literatura, proporcionado avanços no conhecimento técnico-científico. Assim, serão apresentados adiante alguns trabalhos que desenvolveram ensaios acelerados relativos ao ataque por sulfato.

A Tabela 1 apresenta os seis artigos selecionados conforme os critérios estabelecidos referentes aos métodos de ensaios acelerados para avaliação do ataque por sulfato, dentre os quais serão discutidos dois.

Tabela 1. Relação de artigos selecionados sobre ensaios acelerados.

\begin{tabular}{|c|c|}
\hline Pesquisadores & Proposta \\
\hline Khelil et al. (2014) & Investigar testes preliminares inspirados em tratamentos eletroquímicos de reabilitação \\
\hline Huang et al. (2016) & $\begin{array}{l}\text { Investigar o efeito do campo elétrico na deterioração induzida por ataque de sulfato no } \\
\text { concreto. }\end{array}$ \\
\hline Zhou et al. (2016) & $\begin{array}{l}\text { Investigar o desempenho mecânico de colunas de concreto reforçadas com fibras } \\
\text { atacadas por sulfato. }\end{array}$ \\
\hline $\begin{array}{l}\text { Zhutovsky e Hooton } \\
\text { (2017) }\end{array}$ & $\begin{array}{l}\text { Investigar condições adequadas de exposição e métodos de avaliação de deterioração } \\
\text { para testes acelerados. }\end{array}$ \\
\hline Gu et al. (2018) & $\begin{array}{l}\text { Investigar as metodologias existentes de teste acelerado, incluindo: umedecimento e } \\
\text { secagem, e aumento da concentração de ácido sulfúrico }\end{array}$ \\
\hline Yu et al. (2018) & $\begin{array}{c}\text { Investigar o comportamento de amostras de argamassa sob imersão total e ciclos de } \\
\text { molhagem e secagem com solução de } \mathrm{Na}_{2} \mathrm{SO}_{4} \text { a } 0 \% \text { e } 5 \% \text {. }\end{array}$ \\
\hline
\end{tabular}

No estudo realizado por Huang et al. (2016), o objetivo foi investigar o efeito do campo elétrico na deterioração induzida por ataque de sulfato no concreto. O cimento Portland utilizado nesta investigação consistiu em $95 \%$ de clínquer e 5\% de gesso em peso. Foram utilizadas cinzas volantes, calcário em pó e areia siliciosa com o módulo de finura de 2,70 .

Para tal, soluções de $\mathrm{Na}_{2} \mathrm{SO}_{4}(5 \%)$ e de $\mathrm{NaOH}(2,8 \%)$ foram adicionadas às células catódica e anódica, respectivamente. Em seguida, um campo elétrico pulsado de baixa frequência foi empregado, com voltagem de $30 \mathrm{~V}$. O ciclo de pulso foi de 40 segundos, com períodos de circulação e interrupção de 20 segundos. Dessa forma, uma superfície (próxima à solução de sulfato) da amostra também foi exposta no teste de imersão, enquanto as outras superfícies foram seladas com vaselina. O teste de ação combinado de ataque de sulfato e campo elétrico é mostrado esquematicamente na Figura 2 .

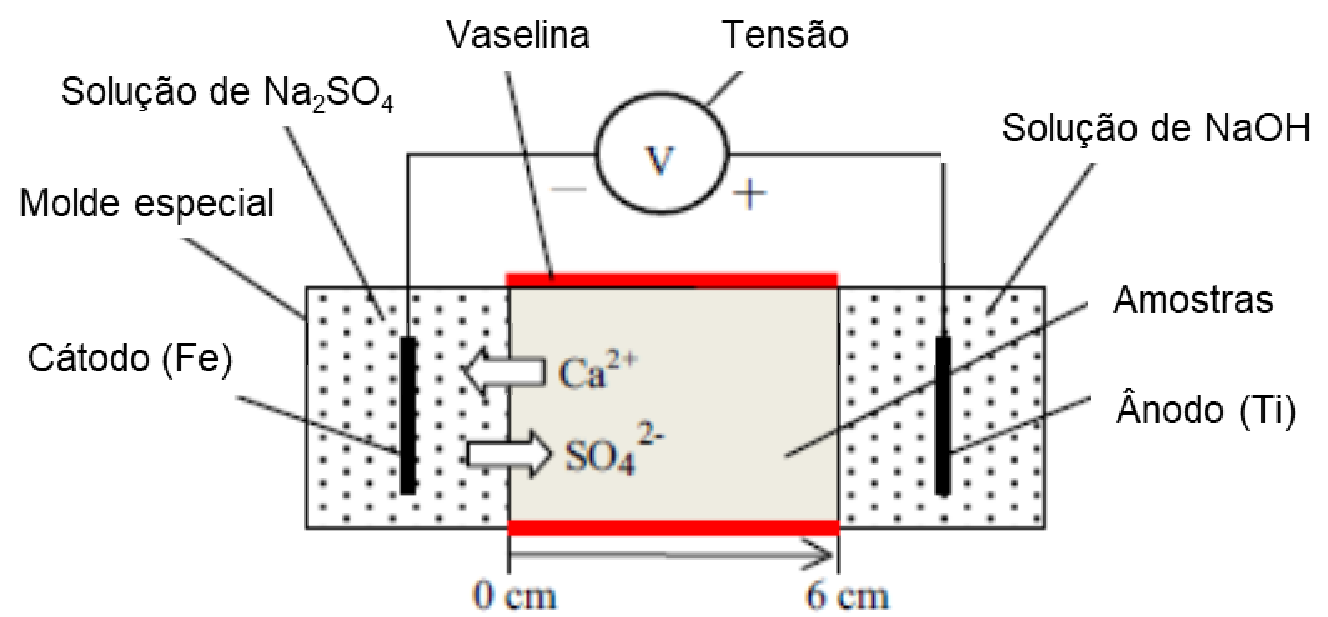

Figura 2 - Esquema de ação combinada para ensaio acelerado (HUANG et al., 2016).

Na Figura 3 apresenta-se o teor de sulfato migrado da argamassa posterior à imersão e às exposições combinadas. Após 90 dias de imersão, a amostra teve um significativo aumento de $\mathrm{SO}_{3}$ perto da superfície exposta, e a profundidade de sulfato e conteúdo máximo de $\mathrm{SO}_{3}$ foi de cerca de $1,0 \mathrm{~cm}$ e $0,84 \%$, respectivamente. No entanto, o teor de sulfato e profundidade após 30 dias de exposição combinada foram significativamente superiores aos da exposição por imersão. Até 90 dias, o conteúdo de $\mathrm{SO}_{3}$ progrediu ainda mais na amostra, com uma concentração máxima de $\mathrm{SO}_{3}$ em torno de $2,84 \%$. A clara diferença dos perfis $\mathrm{SO}_{3}$ entre a imersão e as condições combinadas indicou que o campo elétrico acelerou a migração de íons sulfato na amostra. 


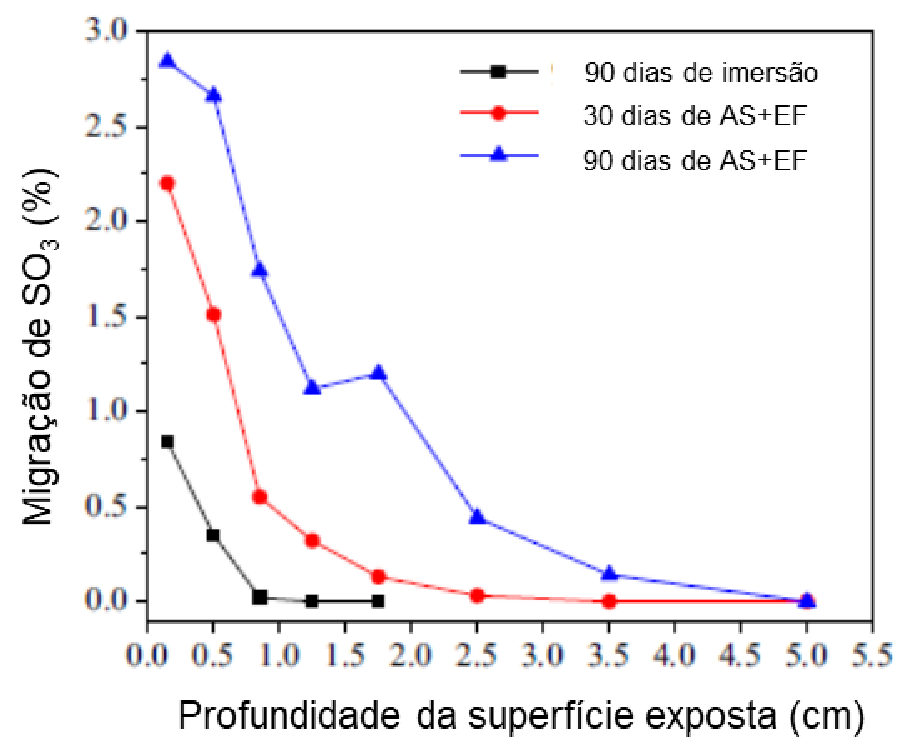

Figura 3 - Gráfico de resultado do ensaio acelerado: Profundidade x $\mathrm{SO}_{3}$ (HUANG et al., 2016).

Diante dos fatos apresentados, os autores concluíram que o campo elétrico acelerou a deterioração induzida por ataque de sulfato e facilitou o ingresso de íons sulfato na matriz de argamassa. Assim o ataque de sulfato combinado com o campo elétrico acelerou o processo de dano da amostra em comparação com o ataque de sulfato por imersão, possibilitando a realização do ensaio com maior velocidade.

No estudo realizado por Zhutovski e Hooton (2017), o objetivo foi avaliar condições adequadas de exposição e métodos de avaliação de deterioração adequados para testes acelerados. Os autores analisaram amostras submetidas à 100 ciclos térmicos entre 5 e $30^{\circ} \mathrm{C}$, onde foi avaliado resistência de amplas misturas de argamassa. As condições de exposição ao ataque por sulfatos envolveram ciclos térmicos das amostras imersas em solução de sulfato de sódio a 30\%. A solução foi substituída a cada 5 ciclos. Para implementar a termo ciclagem, recipientes de plástico selados com as amostras imersas em solução de sulfato de sódio foram colocados em um banho de água programável controlado termicamente. As maiores e menores temperaturas alvo dos ciclos foram estabelecidas em $30^{\circ} \mathrm{C}$ e $5^{\circ} \mathrm{C}$, respectivamente. A duração aproximada de um ciclo térmico completo foi de cerca de $24 \mathrm{~h}$.

Assim, analisou-se a exposição acelerada da resistência física do ataque por sulfatos em argamassas com relação a/c de 0,35, 0,40, 0,45 e 0,50. Ademais, argamassas com substituição parcial de cimento por adições, para a/c de 0,40, também foram avaliadas. As adições empregadas foram: escória de alto forno granulada moída (EAFGM) e cinzas volantes (CV), com dois níveis de substituição de cimento de 45 e $65 \%$, para EAFGM, e 20 e $40 \%$ para CV.

A aparência visual das amostras após 100 ciclos térmicos permitiu observar que as superfícies de todas as amostras mostram evidências de deterioração. A extensão do dano foi classificada com base no tamanho residual da amostra. No entanto, o caráter do dano superficial em todas as amostras é semelhante na aparência.

A perda de massa foi medida usando balanças com capacidade de $3 \mathrm{~kg}$ com sensibilidade de 0,01 gramas. Os resultados obtidos são apresentados em função do número do ciclo térmico, na Figura 4. A variação na resistência ao ataque por sulfatos entre diferentes misturas de argamassa pelo ensaio é evidente, demonstrando grande sensibilidade, bem como a capacidade de quantificar os danos em uma ampla gama de traços. 


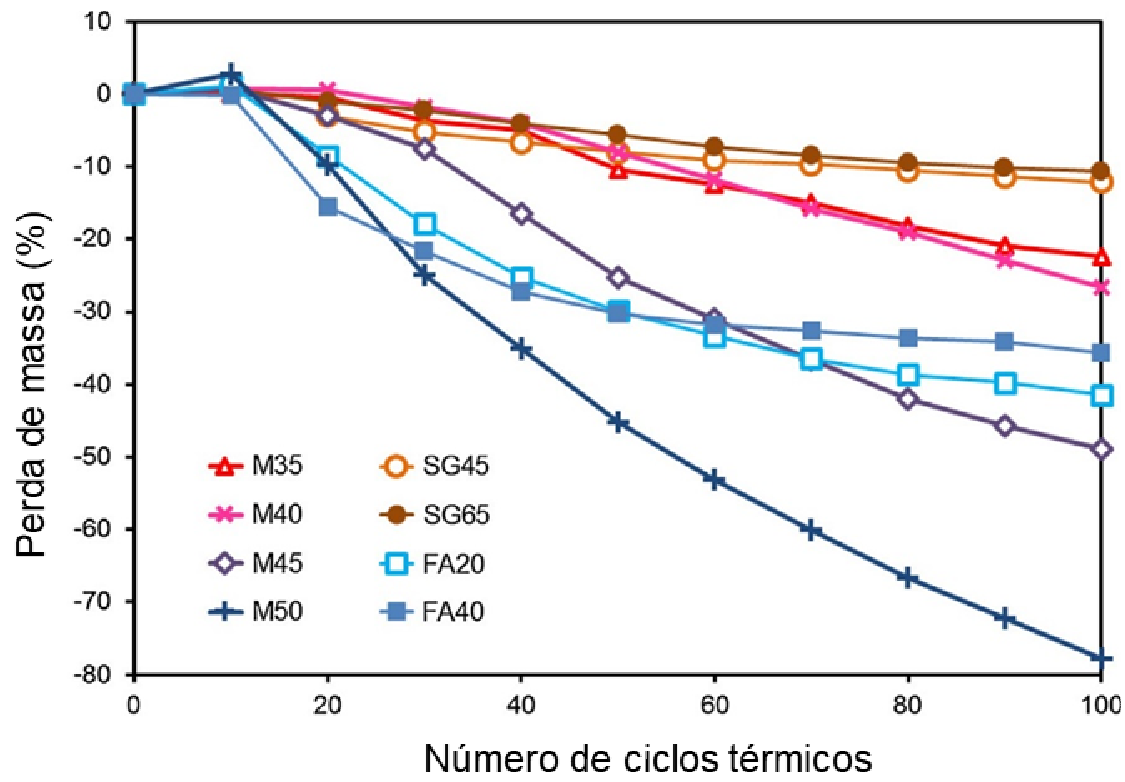

Figura 4 - Perda de massa em função do número de ciclos térmicos (ZHUTOVSKI e HOOTON, 2017).

O estudo de Zhutovski e Hooton (2017) confirmou que a ciclagem térmica em solução de sulfato de sódio a $30 \%$ mostrou ser uma boa exposição para testes acelerados da resistência ao ataque por sulfatos para uma ampla gama de projetos de mistura de argamassa. O método mais sensível e eficiente para a avaliação da taxa de deterioração e extensão dos danos na exposição do ataque por sulfatos foi a perda de massa.

\subsection{Modelagem de ataque interno}

A Tabela 2 apresenta os seis artigos selecionados conforme os critérios estabelecidos respectivos aos métodos empregados para a modelagem do ataque interno por sulfatos (ISA: Internal sulfate attack). Enfatiza-se aqui que há pouca literatura acerca do presente tema, diferentemente do que ocorre para o ataque externo, conforme será apresentado adiante. Assim, poucos foram os modelos encontrados para o ISA, limitando-se aos artigos que seguem, dentre os quais serão discutidos três.

Tabela 2. Relação de modelos de ataque interno por sulfatos (ISA) selecionados.

\begin{tabular}{|c|c|}
\hline Pesquisadores & Proposta \\
\hline Oliveira et al. (2013) & $\begin{array}{l}\text { Modelo numérico, cinético e simplificado para quantificar a expansão devido à oxidação } \\
\text { do sulfeto de ferro e à formação de etringita secundária. }\end{array}$ \\
\hline Campos et al. (2016a) & $\begin{array}{l}\text { Modelo de difusão para quantificar a expansão devido à oxidação da pirrotita nos } \\
\text { agregados. }\end{array}$ \\
\hline Chen et al. (2016) & $\begin{array}{l}\text { Modelo químico-mecânico para avaliar o dano pela corrosão e pela formação de etringita } \\
\text { tardia, devido ao ISA. }\end{array}$ \\
\hline Campos et al. (2016b) & $\begin{array}{c}\text { Validação do modelo de Campos et al. (2016a) por meio de estudo de caso de uma } \\
\text { barragem na Espanha. }\end{array}$ \\
\hline
\end{tabular}

Em estudo realizado por Oliveira et al. (2013), são propostas equações para quantificar a expansão devido à oxidação de sulfeto de ferro e à formação de etringita secundária no concreto. Dessa forma, foi criado um modelo simplificado para avaliar a evolução da expansão, o qual permite uma previsão direta das expansões geradas pelo ISA, levando em conta as características físicas e químicas do concreto e do agregado. A expansão volumétrica do concreto calculada com este modelo foi utilizada para um estudo de caso, com intuito de estimar as variações dimensionais em uma barragem.

O modelo do parágrafo anterior também pode ser utilizado para a previsão de movimentos futuros da estrutura. Ao final, o resultado obtido se encaixou nas aferições realizadas e, portanto, é válido para a previsão de movimentos futuros da estrutura.

A Equação 1 apresenta a fórmula para o cálculo da expansão real total $\left(\Delta \mathrm{V}_{T}, \mathrm{r}\right)$, que é função da expansão potencial total $\left(\Delta \mathrm{V}_{\mathrm{T}}\right)$. Porém, esta expansão potencial só é alcançável caso todos os produtos de oxidação de etringita e pirrotita 
secundários gerarem expansão na pasta de cimento. Contudo, na prática, os produtos expansivos também ocupam os vazios presentes no concreto, para o qual se introduz um parâmetro de amortecimento ( $\alpha$ ) - Equação 2 - o qual estima a quantidade que realmente causará expansão do elemento.

$$
\begin{aligned}
& \Delta V_{T}, r=\alpha \cdot \Delta V_{T} \\
& \alpha=e^{(-0,24 \cdot \varphi)}
\end{aligned}
$$

Todavia, para o cálculo da deformação do concreto devido ao ISA ao longo do tempo, a expansão real total estimada na Equação 1 não é suficiente. É importante, também, conhecer a cinética da reação que controla o fenômeno expansivo. Considerando que as partículas de diferentes diâmetros oxidam simultaneamente, o estudo de OLIVEIRA et al. (2013) desenvolveu um procedimento para somar a oxidação de cada grão para obter a cinética de oxidação global de todos os agregados. A formulação ajustada é apresentada na Equação 3, sendo alguns de seus parâmetros determinados pelas Equações 4 a 7, onde G1 e G2 são determinados por regressão linear a partir da curva granulométrica do agregado e D é o coeficiente de difusão do oxigênio. Finalmente, a Equação 8 apresenta a fórmula para o cálculo da expansão volumétrica.

$$
\begin{gathered}
\% R=\frac{t_{e q}}{\left(A+B \cdot t_{e q}+C \cdot \sqrt{t_{e q}}\right)} \\
t_{e q}=t \cdot \frac{C}{(0,26)} \\
A=\frac{\left(3,29 \cdot G_{2}^{-1}-24,51 e^{\left(\frac{-0,13}{G_{1}}\right)}+29,81 \cdot G_{1}\right)}{98,07 \cdot 10^{4} \cdot D} \\
C=\frac{\left(38 \cdot e^{\left(\frac{0,31}{G_{1}}-20,4 \cdot G_{2}\right)}+0,58 \cdot G_{2}\right) \cdot D^{-0,5}}{10^{2}} \\
\mathcal{E}_{V}=\Delta V_{T}, r \cdot \% R
\end{gathered}
$$

No estudo realizado por Campos et al. (2016a), o modelo de difusão é desenvolvido para o problema do ataque interno por sulfato (ISA) devido à oxidação da pirrotita nos agregados, calculando a expansão volumétrica ou linear resultante do fenômeno. O modelo leva em consideração a cinética da oxidação da pirrotita sob os dois principais oxidantes e o transporte de oxigênio no concreto, mas não considera a variação de $\mathrm{pH}$. A dependência do teor de água do concreto é incluída na análise, que afeta a cinética da reação e a difusão do oxigênio. O modelo desenvolvido é aplicado a duas barragens reais afetadas pelo ISA.

A ISA desenvolve-se em duas etapas: uma reação primária devido a oxidação dos su-fetos de ferro no agregado (pirrotita) e um reação secundária devido a um ataque de sulfato dos produtos da oxidação à pasta de cimento. A reação primária é abordada por meio de uma reação de difusão no modelo que leva em conta a cinética da oxidação, reações da pirrotita e a difusão de oxigênio no concreto. A evolução do processo depende da oxidação da pirrotita em agregados.

A pirrotita é oxidada quando exposta ao oxigênio e na presença de água, levando à formação de sulfatos e hidróxidos de ferro, assim causando uma diminuição na alcalinidade do concreto. A oxidação destes sulfatos pode ser iniciada pela atmosfera ou íons de ferro. 
As equações empregadas pelo modelo de Campos et al. (2016a) são apresentadas a seguir (Equações 9 a 11), onde $\Delta \mathrm{V} / \mathrm{V}_{(\mathrm{t})}$ é a evolução da expansão volumétrica, $\Delta \mathrm{V} / \mathrm{V}_{(\infty)}$ a expansão total, $\left[\mathrm{Fe}_{1-\mathrm{x}} \mathrm{S}\right]_{(0)}$ a concentração inicial de pirrotita e $\left[\mathrm{Fe}_{1-\mathrm{x}} \mathrm{S}\right]_{(\mathrm{t})}$ a concentração num dado tempo t. Ademais, $\mathrm{m}_{\mathrm{x}}$ é o volume molar, $\mathrm{w}_{\mathrm{i}} \mathrm{o}$ coeficiente estequiométrico, $\phi$ o teor de porosidade e $f$ a porosidade capilar.

$$
\begin{gathered}
\left(\frac{\Delta V}{V}\right)_{(t)}=\left(\frac{\Delta V}{V}\right)_{\infty} \cdot\left(\frac{\left[F e_{1}-{ }_{x} S\right]_{(0)}-\left[F e_{1}-{ }_{x} S\right]_{(t)}}{\left[F e_{1}-{ }_{x} S\right]_{(0)}}\right) \\
\left(\frac{\Delta V}{V}\right)_{\infty}=\left(\frac{\Delta V}{V}\right)_{\text {total }}-f \cdot \phi \\
\left(\frac{\Delta V}{V}\right)_{\text {total }}=\left(\frac{\Delta V}{V}\right)_{F e(O H)_{3}}+\left(\frac{\Delta V}{V}\right)_{\text {etringita }}
\end{gathered}
$$

Considerando a importância do efeito da água e da temperatura sobre os efeitos da difusão do oxigênio e a cinética da difusão, é proposto um método indireto de levar em conta tal fator sobre os coeficientes de difusão. A Equação 12 apresenta tal influência, calculando o coeficiente cinético $\left(\mathrm{k}_{\mathrm{i}(0)}\right)$, onde $\mathrm{k}_{\mathrm{i}(1)}$ é o coeficiente cinético da reação i no concreto saturado; $\theta$ o teor de água; $\beta$ é um parâmetro que controla a forma da curva, obtido conforme Figura 5. Por outro lado, a Equação 13 apresenta o efeito da água sobre o coeficiente de difusão do oxigênio, onde $\mathrm{D}_{(0)}$ é o coeficiente de difusão do oxigênio e $\gamma$ é um parâmetro que governa a forma da curva.

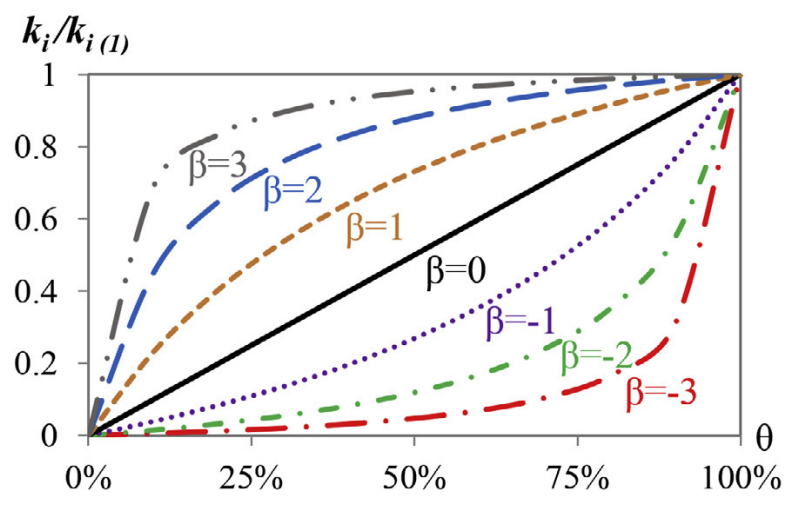

Figura 5 - Variação do coeficiente cinético das reações com o teor de água do concreto (CAMPOS et al., 2016a).

$$
\begin{gathered}
k_{i(\theta)}=k_{i(1)} \cdot\left[\frac{\theta e^{\beta}}{1+\theta \cdot\left(e^{\beta}-1\right)}\right] \\
D_{(\theta)}=D_{(0)} \cdot\left[1-\frac{\theta e^{\gamma}}{1+\theta \cdot\left(e^{\gamma}-1\right)}\right]
\end{gathered}
$$

O modelo proposto foi aplicado em duas barragens reais, de forma a compreender o seu potencial, que poderia prever a evolução ao longo do tempo e a distribuição das deformações na estrutura devido ao ISA. Os resultados obtidos ao final do estudo validam o modelo e demonstram seu potencial para avaliar e prever o comportamento das estruturas afetadas pelo ISA.

Aplicando os conceitos desse estudo, Campos et al. (2016b) estudaram - dois anos depois - uma barragem de gravidade na Espanha. Análises prévias realizadas revelaram a presença de sulfetos de ferro nos agregados e etringita secundária na interface do agregado com a pasta. Assim, considerou-se o ataque interno de sulfato como a principal causa de danos. A disponibilidade de água para ocorrência da reação foi analisada, pois em caso de pouca água no ambiente das partículas de pirrotita, a oxidação não ocorre. Por outro lado, aumentar o teor de água aumenta o número de partículas 
de pirrotita em contato com a água, apresentando condições para o desenvolvimento da oxidação. Ressalta-se que a difusão do $\mathrm{Fe}^{2+}$ e do $\mathrm{Fe}^{3+}$ não foi levada em consideração, porque o modelo proposto considera o concreto como homogêneo. Finalmente, por meio da mesma equação empregada no estudo anterior dos mesmos autores, a evolução da expansão volumétrica ao longo do tempo foi calculada e expressa como função da oxidação da pirrotita, conforme Equação 7. Os resultados confirmaram o diagnóstico proposto e a capacidade do modelo em reproduzir o comportamento da barragem.

\subsection{Modelagem de ataque externo}

A Tabela 3 apresenta sete artigos selecionados com os respectivos métodos empregados para a modelagem do ataque externo por sulfatos (ESA). Destaca-se que, para esse tipo de ataque, a literatura possui uma diversidade maior de estudos em comparação ao ISA. Tal fato se deve provavelmente à maior complexidade deste último em relação ao ataque externo. Dessa forma, diversos modelos foram encontrados para o fenômeno referente ao presente item, os quais foram filtrados através dos critérios estabelecidos no item 2, dentre os quais será discutido um.

No estudo realizado por Soive et al. (2016), comparou-se um modelo numérico físico-químico com dados experimentais obtidos em uma pasta de cimento Portland exposta ao ataque por sulfato externo por meio de análises experimentais. Para tal, empregou-se análises de Microscopia Eletrônica de Varredura (MEV) e Microtomogarafia (XRadia MicroXCT-400) que forneceram perfis de concentração total de íons para uma determinada amostra.

Tabela 3. Relação de modelos sobre ataque externo por sulfatos selecionados.

\begin{tabular}{|c|c|}
\hline Pesquisadores & Proposta \\
\hline Ikumi et al. (2014) & $\begin{array}{c}\text { Modelo numérico de difusão alternativo mais realista, considerando o efeito dos poros e } \\
\text { constituição dos materiais. }\end{array}$ \\
\hline Yu et al. (2015) & $\begin{array}{c}\text { Modelo de elementos finitos para simulação da degradação, incluindo três submodelos } \\
\text { principais: difusão iônica, reação química e quantificação de danos. }\end{array}$ \\
\hline Soive et al. (2016) & $\begin{array}{c}\text { Modelo numérico físico-químico, comparado dados experimentais para validação do } \\
\text { método. }\end{array}$ \\
\hline Ikumi et al. (2016) & $\begin{array}{c}\text { Modelo físico-químico simplificado para avaliação de dano potencial em estruturas } \\
\text { expostas a ambientes agressivos. }\end{array}$ \\
\hline Soive e Tran (2017) & $\begin{array}{c}\text { Modelagem físico-química numérica considerando a cinética da dissolução/precipitação } \\
\text { e a complexidade superficial. }\end{array}$ \\
\hline Cefis e Comi (2017) & $\begin{array}{c}\text { Modelagem numérica físico-química das consequências mecânicas sob condições } \\
\text { parcialmente ou totalmente saturadas. }\end{array}$ \\
\hline Yu e Zhang (2018) & $\begin{array}{c}\text { Modelo numérico integrado para avaliação da deterioração das propriedades mecânicas } \\
\text { de argamassas. }\end{array}$ \\
\hline
\end{tabular}

Os ensaios foram realizados com duas amostras de relação água/cimento 0,4, com corpos de prova cilíndricos de 160 $\mathrm{mm}$ de altura por $20 \mathrm{~mm}$ de diâmetro, sendo imersas durante 160 dias em 1,7 litros de solução de $\mathrm{Na}_{2} \mathrm{SO}_{4}$ (concentração de sulfato de $3 \mathrm{~g} / \mathrm{L}$ ), após 28 dias de cura com água. As amostras foram revestidas em suas duas extremidades (superior e inferior) com uma resina de vinil à prova de água, sendo expostas ao ataque de sulfato somente em suas superfícies circunferenciais.

Quanto às modelagens empregadas no estudo, a hidratação da pasta de cimento foi explorada usando um método de minimização da Bureau de Recherches Géologiques et Minières (BRGM), o Serviço Geológico da França. Quanto aos mecanismos de transporte, a difusão foi considerada supostamente como principal fenômeno. Para tal, fez-se uso de um modelo de difusão fickiana multiespécies, conforme abordagem da Nernst-Planck. Ademais, os fenômenos de sorção (adsorção e absorção) são considerados puramente químicos e modelados pela parte geoquímica da simulação. A Equação14 e a Equação 15 apresentam o modelo adotado, tal que: $c_{i}$ é a concentração; $D_{e, i}$ é o coeficiente de difusão efetivo no meio poroso, que é função da tortuosidade $(\tau)$, porosidade $(\theta)$ e coeficiente de difusão puro em água $\left(D_{w, i}\right)$; e $\Delta_{\mathrm{ci}}$ é o operador de divergência.

$$
\begin{aligned}
& \frac{\partial C_{i}}{\partial t}=D_{e}, i \cdot \Delta C_{i} \\
& D_{e, i}=\tau \cdot \theta \cdot D_{w, i}
\end{aligned}
$$


Os autores empregaram, para a associação de modelos, o procedimento usado por Wissmeier e Barry (2011). Assim, a parte de transporte de soluto é calculada pelo software COMSOL - que é um Microsoft Component Object Model $(\mathrm{COM})$ - e as reações geoquímicas são calculadas pelo software PHREEQC.

O modelo testado conseguiu calcular a posição da frente de precipitação da etringita e a posição da primeira diminuição devido à dissolução da portlandita, enquanto a lixiviação de portlandita e etringita não foi avaliada com precisão nos primeiros milímetros devido à necessidade de levar em consideração alteração das propriedades de transporte na zona degradada. Pelo estudo numérico paramétrico realizado, constatou-se o efeito de aceleração pela renovação permanente da solução. Ademais, a influência do controle de $\mathrm{pH}$ também depende da renovação da solução; já a presença de $\mathrm{CO}_{2}$ não desempenhou um papel significativo. Os resultados obtidos pelos ensaios empregados pelo estudo experimental, em comparação à modelagem, são apresentados na Figura 6 e na Figura 7.

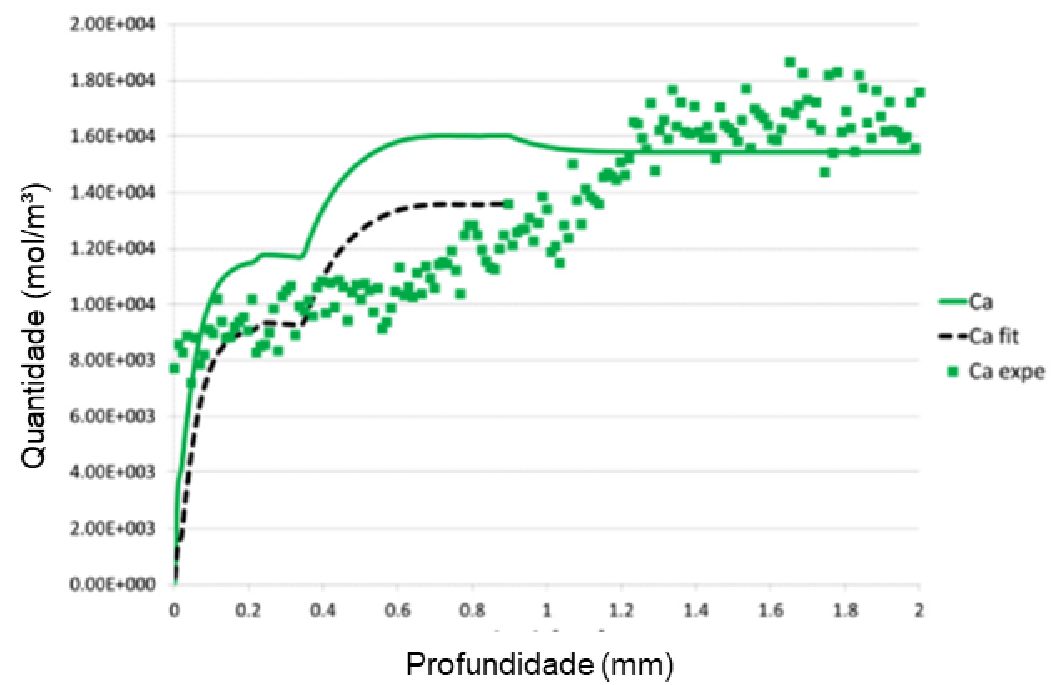

Figura 6 - Comparação entre os perfis de Ca numéricos experimentais e numéricos após 160 dias (SOIVE et al., 2016).

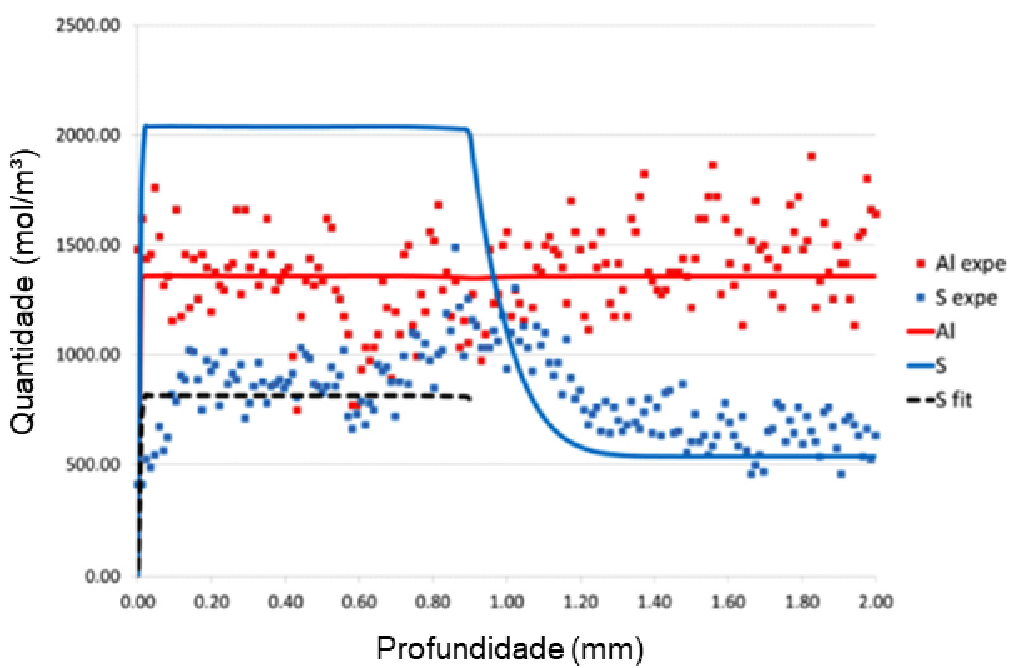

Figura 7 - Comparação entre os perfis de AI e S numéricos experimentais e numéricos após 160 dias (SOIVE et al., 2016). 


\section{CONCLUSÕES}

Tendo em vista os aspectos observados, pode-se concluir que, dentre os fatores que influenciam o ataque por sulfatos, alguns são intrínsecos ao material em estudo, como relação água/cimento, tipo de cimento e adições, e outros são influenciados por fatores externos como, tipo e tempo de cura, concentração de sulfatos, tempo de exposição e tamanho das amostras. Para tal, diversos modelos de ensaios acelerados são propostos na literatura, além dos já padronizados e normatizados, tentando compreender os fenômenos envolvidos no processo, de modo a controlar as variáveis presentes.

Analisando os fatores de modelagem de ataque por sulfatos e ensaios acelerados, destaca-se a importância de todos os modelos serem validados para diversas situações, além das que foram adotadas para o seu desenvolvimento. Assim, bons modelos devem resultar em dados que remetam à realidade, de modo a representar de maneira mais fiel o que deverá ocorrer ao longo da vida útil do material estudado.

O mesmo vale para os ensaios acelerados, tal que seja possível verificar o comportamento da amostra em situações reais por meio de correlações com os métodos aplicados. Assim para realizar uma análise dos resultados obtidos, o ideal é definir um espaço amostral coerente, buscando uma padronização das amostras analisadas, onde o ensaio deve responder aos questionamentos advindos da pesquisa.

Para os resultados obtidos no presente artigo de revisão, constatou-se a presença de maior volume de pesquisas voltadas ao ataque externo, tanto para ensaios quanto para modelagem. Acredita-se que tal fato se deve à complexidade envolvida nos fenômenos internos e, também, à maior preocupação existente quanto ao ataque externo, que é resultado de condições de exposição do material, sendo que dificilmente podem ser evitadas, causando grandes degradações aos concretos.

Mediante ao que foi apresentado, observa-se que as principais áreas de pesquisa que precisam avançar para o desenvolvimento futuro desta linha temática são os estudos do ataque interno por sulfatos, principalmente com a padronização de um ensaio acelerado. Outro ponto a destacar é reduzir a complexidade dos modelos para prever o ataque, de maneira a estimular a sua aplicação prática em situações corriqueiras da construção civil.

\section{AGRADECIMENTOS}

Os autores agradecem a Agência Nacional de Águas (ANA), CAPES, CNPq, Fundação Araucária, ao Departamento de Construção Civil e ao Programa de Pós-Graduação em Engenharia de Construção Civil (PPGECC) da UFPR.

\section{REFERÊNCIAS}

CAMPOS et al. Diffusion-reaction model for the internal sulfate attack in concrete. Construction and Building Materials, 102:531-540, 2016.

CAMPOS et al. Effects of an internal sulfate attack and an alkali-aggregate reaction in a concrete dam. Construction and Building Materials, 166:668-683, 2016.

CEFIS, N.; COMI, C. Chemo-mechanical modelling of the external sulfate attack in concrete. Cement and Concrete Research, 93:57-70, 2017.

CHEN et al. A new chemo-mechanical model of damage in concrete under sulfate attack. Construction and Building Materials, 115:536-543, 2016.

COUTINHO, J. S. Ataque por Sulfatos. Faculdade de Engenharia do Porto. Porto. 2001.

GU et al. Evaluation of accelerated degradation test methods for cementitious composites subject to sulfuric acid attack; application to conventional and alkali-activated concretes. Cement and Concrete Composites, 87:187-204, 2018.

HOPPE FILHO, J. et al. Ataque de matrizes cimentícias por sulfato de sódio: adições minerais como agentes mitigadores. Cerâmica, 61:168-177, 2015.

HUANG et al. Deterioration of mortars exposed to sulfate attack under electrical field. Construction and Building Materials, 117:121-128, 2016. 
IKUMI et al. Alternative methodology to consider damage and expansions in external sulfate attack modeling. Cement and Concrete Research, 63:105-116, 2014.

IKUMI et al. Simplified methodology to evaluate the external sulfate attack in concrete structures. Materials and Design, 89:1147-1160, 2016.

KHELIL, N.; AUBERT, J. E.; ESCADEILLAS; G. Development of an accelerated test for Internal Sulfate Attack study. MATEC Web of Conferences, 11:01042, 2014.

MEHTA, P. K.; MONTEIRO, P. J. M. Concreto: Estrutura, propriedades e materiais. São Paulo. Pini. 2014.

OLIVEIRA et al. New kinetic model to quantify the internal sulfate attack in concrete. Cement and Concrete Research, 43:95-104, 2013.

SOIVE et al. Parametrical study of the cementitious materials degradation under external sulfate attack through numerical modeling. Construction and Building Materials, 112:267-275, 2016.

SOIVE, A.; TRAN, V. Q. External sulfate attack of cementitious materials: New insights gained through numerical modeling including dissolution/precipitation kinetics and surface complexation. Cement and Concrete Composites, 83:263-272, 2017.

WISSMEIER, L.; BARRY, D. A. Simulation tool for variably saturated flow with comprehensive geochemical reactions in two- and three-dimensional domains. Environmental Modelling \& Software, 26:210-218, 2011.

YU et al. Behavior of mortar exposed to different exposure conditions of sulfate attack. Ocean Engineering, 157:1-12, 2018.

YU et al. Numerical modelling of degradation of cement-based materials under leaching and external sulfate attack. Computers and Structures, 158:1-14, 2015.

YU, Y.; ZHANG, Y. X. Numerical modelling of mechanical deterioration of cement mortar under external sulfate attack. Construction and Building Materials, 158:490-502, 2018.

ZHOU et al. Effect of sulfate attack on the stress-strain relationship of FRP-confined concrete. Construction and Building Material, 110:235-250, 2016.

ZHUTOVSKY, S.; HOOTON, R. D. Accelerated testing of cementitious materials for resistance to physical sulfate attack. Construction and Building Materials, 145:98-106, 2017. 